\title{
Design, Simulation and Experiment of a Cusp Electron Beam for Millimeter Wave Gyro-devices
}

\author{
Wenlong He, Craig R. Donaldson, Fengping Li, Adrian W. Cross, Alan D.R. Phelps, \\ Kevin Ronald, Craig W. Robertson, Colin G. Whyte and Liang Zhang \\ SUPA, Department of Physics, University of Strathclyde, Glasgow, G4 ONG, Scotland, UK \\ w.he@strath.ac.uk
}

\begin{abstract}
The design, simulation and experiment of a thermionic cusp electron gun that is to be used for millimeter wave generation will be presented. A cusp gun uses a nonadiabatic magnetic field reversal to obtain azimuthal motion on an electron beam resulting in an annular shaped, axisencircling beam. The cusp gun was designed to generate a beam of $1.5 \mathrm{~A}$ at $40 \mathrm{kV}$ with an adjustable velocity ratio of up to 3.0. The beam had a simulated axial velocity spread of $7.4 \%$ and alpha spread of $10.1 \%$. The beam had an averaged radius of $0.35 \mathrm{~mm}$ and beam thickness of $0.05 \mathrm{~mm}$ which is ideal to drive sub-mm wave gyro-devices under investigation.
\end{abstract}

Keywords: Cusp; electron gun; millimetre wave gyrodevice; gyrotron.

\section{Introduction}

Gyro-devices are promising candidates for the generation of high power, high frequency, coherent radiation at wavelengths in the millimetre to sub-millimetre range. A few projects combining theoretical, numerical and experimental study of such devices are underway to generate or amplify radiation in this frequency range required for a number of applications. A W-Band Gyrotron Backward Wave Oscillator (gyro-BWO) based on a helically corrugated waveguide is simulated to achieve a $3 \mathrm{~dB}$ frequency tuning band of $\sim 84-104$ $\mathrm{GHz}$, output power of $\sim 10 \mathrm{~kW}[1,2]$. A harmonic gyrotron operated at the $7^{\text {th }}$ harmonic of the electron cyclotron mode and with a $\mathrm{TE}_{71}$ output mode has been designed to achieve $\sim 500 \mathrm{~W}$ at $\sim 390 \mathrm{GHz}[3]$. At the same time a W-band Gyrotron Traveling Wave Amplifier (Gyro-TWA) based on a helically corrugated waveguide is under design to achieve a maximum output power of $\sim 5 \mathrm{~kW}$ with a $3 \mathrm{~dB}$ bandwidth of 90-100GHz [4].

Presented in this paper are the design, simulation and latest experimental results of a novel cusp electron beam source to drive the beam-wave interactions in the aforementioned Gyro-devices. This electron gun is required to produce an axis-encircling, annular shaped electron beam of current $1.5 \mathrm{~A}$, energy $40 \mathrm{keV}$ and a pitch alpha of up to 3 with the beam optimized for interaction in the cavities. The pitch alphas (velocity ratio $\alpha$ ) of the electron beam generated by the cusp gun are chosen to be 1.65 and 1.2 respectively when driving the W-band gyro-BWO and gyro-TWA. The interaction between the second harmonic cyclotron beam mode and the $\mathrm{TE}_{21}$ like eigenwave based on the CRM instability allows broadband microwave growth to be achieved both in the gyro-BWO and gyro-TWA. An alpha value of 3 can be obtained to drive the high harmonic $\mathrm{TE}_{71}$ mode gyrotron with an output frequency of $390 \mathrm{GHz}$. When using a weakly relativistic electron beam the interaction coefficient is significantly reduced for the high harmonic cyclotron mode of operation. A high $\alpha$ can lower the starting current of the operating mode and lead to higher efficiency.

The cusp electron gun is a novel way of creating an axisencircling annular electron beam ideal for use in harmonic gyro-devices. Conventional electron guns mainly used in gyro-devices are the Magnetron Injection Gun (MIG) and Pierce gun. Mode selectivity is poor in the MIG as the coupling for such a beam is strongest with the fundamental mode. In the Pierce gun case, a pencil line electron beam is kicked into an axis-encircling path. However, for this CW operation the Pierce gun would not be suitable as the kicker magnet is feasible primarily in pulsed mode. Due to the high power of the spent electron beam, an annular shaped electron beam is desirable for beam energy recovery using a depressed collector method because of the reduced power density on the collecting electrodes.

\section{Design}

A 'smooth' cusp formed by two coils without any magnetic shaping poles was used to generate the required axisencircling electron beam because in this case the Larmor step is much larger due to the small amplitude of the cusp and small transverse velocity component (Fig. 1). This allows a field reversal over a longer distance.[5]

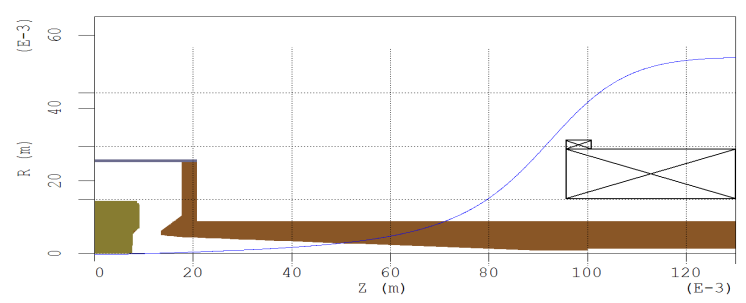

Fig. 1 A schematic showing the cusp gun, its magnetic coils and overlaid magnetic field profile.

Due to azimuthal symmetry of the magnetic and electric field, conservation of the electron canonical angular momentum [6] gives rise to an axis encircling beam. The electrons gain transverse velocity mainly due to $V_{z} \times B_{r}$ force in the cusp region. It is possible to show that the alpha value of the beam 
in the downstream uniform B field region is governed by Eq. (1):

$$
\alpha=\frac{V_{\perp}}{V_{z}}=\sqrt{\frac{-r_{c}{ }^{2} \omega_{c} \omega_{0}}{V^{2}+r_{c}{ }^{2} \omega_{c} \omega_{0}}}
$$

Here $V$ and $\omega$ are electron velocity and cyclotron frequency, subscripts ' $\perp$ ', ' $\mathrm{z}$ ' denote transverse and $\mathrm{z}$ components, ' 0 ' and 'c' the final and cathode region respectively.

\section{Simulations}

The 3D PiC code Magic was used to simulate and optimize the design of the cusp electron beam source. The geometry of the cusp gun including its magnetic field profile is shown in Fig. 1. In the simulation, the position, size and current of the two coils, the shape and dimension of the focusing electrode, the gap distance between the cathode and anode and the shape of the anode could be adjusted to optimize the beam quality in the downstream region. The emitted beam current, transported current and electron energy, velocity, pitch alpha and its spread were simulated and monitored at a position in the downstream uniform B-field region.

The simulated beam trajectories are shown in Fig. 2. It clearly shows that an axis-encircling beam was obtained.

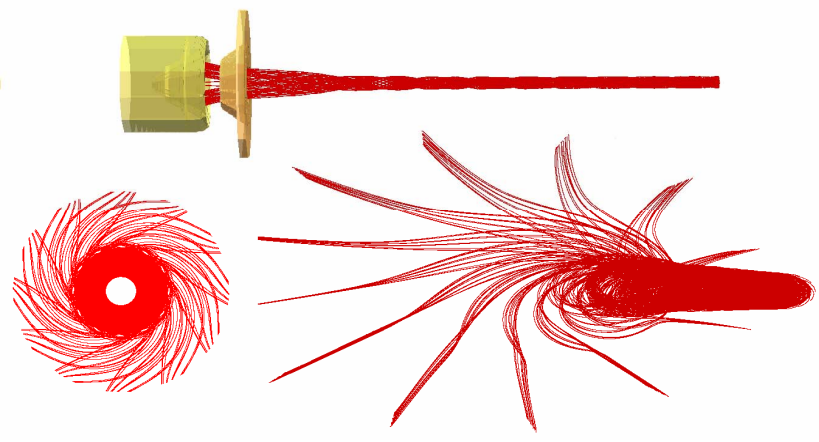

Fig. 2 Simulation results showing the shape and trajectories of the electron beam from the cusp gun.

The alpha value of the electron beam can be adjusted by adjusting the magnetic field amplitude at the cathode or/and at the downstream uniform region as governed by Eq. (1). Simulation predicted the large orbit electron beam has an inner and outer diameter of $0.6 \mathrm{~mm}$ and $0.7 \mathrm{~mm}$ at an alpha value of 1.65 when in the downstream, uniform magnetic field region $\left(\mathrm{B}_{0}=2.1 \mathrm{~T}\right)$. The spread of the alpha is $\sim 10 \%$ as shown in Fig. 3.

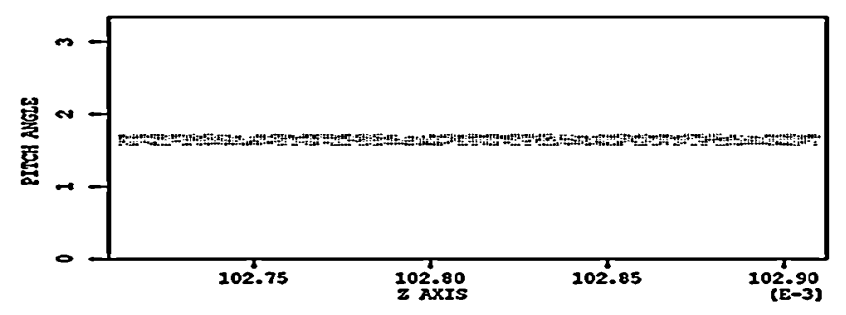

Fig. 3 Velocity pitch angle of the cusp beam.

\section{Experiment}

The construction of the cusp electron gun and various components including magnetic coils, cathode and diode are completed. The diagnostics of the electron beam including a fast, high voltage divider and a Faraday cup for beam voltage and current measurements are designed as well. A scintillator method will be used to measure the beam cross-sectional shape and size hence the velocity ratio $\alpha$. The experimental measurements will be given and compared with the theoretical and simulation results.

\section{Conclusion}

Optimized design for a cusp electron gun for use in sub-mm Gyro-devices is presented. The cusp gun was designed to generate a beam of $1.5 \mathrm{~A}$ at $40 \mathrm{kV}$ with an adjustable velocity ratio of up to 3.0. The beam had a simulated axial velocity spread of $7.4 \%$ and alpha spread of $10 \%$. The beam had inner and outer diameters of $0.6 \mathrm{~mm}$ and $0.7 \mathrm{~mm}$ at an alpha value of 1.65 which is ideal to drive sub-mm wave gyro-devices under investigation.

\section{Acknowledgments}

The authors would like to thank UK EPSRC and the Scottish Universities Physics Alliance (SUPA) and the Overseas Research Students Awards Scheme (ORSAS) for support of this work.

\section{Reference}

[1] W. He, A. D. R. Phelps, C. R. Donaldson, A. W. Cross, K. Ronald, "A W-band Gyro-BWO based on helically corrugated waveguide", Digest of the 32nd Int. Conf. on Infrared and Millimeter Waves, Cardiff, UK, 2007.

[2] C. R. Donaldson, W. He, A. D. R. Phelps, A. W. Cross, K. Ronald, A.R. Young, "The design and simulation of a Wband gyro-BWO", Digest of the $34^{\text {th }}$ IEEE Pulsed Power and Plasma Science Conference, Albuquerque USA, 2007.

[3] F. Li, W. He, C. R. Donaldson, A. D. R. Phelps, A. W. Cross, et al, "The Simulation of an High Power $390 \mathrm{GHz}$ Large-orbit Harmonic Gyrotron", Digest of the International Vacuum Electronics conference, Monterey, USA, 2008.

[4] W. He, A. D. R. Phelps, A. W. Cross, et al, "A W-band Gyrotron Travelling Wave Amplifier Using a Helically Corrugated Waveguide", Digest of the 17th High-Power Particle Beams Conference, Xi'an, P. R. China, 2008.

[5] W. He, C. G. Whyte, E. G. Rafferty, et al, "Axisencircling electron beam generation using a smooth magnetic cusp for gyrodevices", Appl. Phys. Letts, 93, 121591, 2008.

[6] M. J. Rhee and W. W. Destler, "Relativistic electron dynamics in a cusped magnetic field", The Physics of Fluids, 17, No. 8, 1574 (1974). 